\title{
Caracterização morfométrica da Curitiba prismatica
}

\author{
Edson Gardin $\mathbb{1}^{1 *}$ Luciano Farinha Watzlawick $\mathbb{1}^{1}$ Patricia Carla Giloni-Lima $\mathbb{(}^{1}$
} ${ }^{1}$ Universidade Estadual do Centro-Oeste, Alameda Élio Antonio Dalla Vecchia, 838, VilaCarli, Campus Cedeteg, CEP 85040-167,
Guarapuava, PR, Brasil

\begin{abstract}
Original Article
*Corresponding author: gardin@unicentro.br

Palavras-chave:

Sistema faxinal

Floresta Ombrófila Mista

Biometria

Keywords:

Faxinal system

Potential failure

Risk analysis

Received in

2019/04/04

RESUMO: A morfometria da Curitiba prismatica (D. Legrand) Salywon e Landrum foi avaliada em um fragmento de Floresta Ombrófila Mista em sistema faxinal, no município de Rebouças-PR. Foram incluídas as árvores com DAP maior ou igual a $10 \mathrm{~cm}$, nas quais as análises foram realizadas de forma agrupada e por classes diamétricas. A coleta dos dados ocorreu em 2017, sendo medido o DAP, a altura total, a altura de inserção da copa e os quatro raios de projeção de copa. Com esses dados foram calculadas as variáveis morfométricas: diâmetro da copa, comprimento de copa, proporção de copa, grau de esbeltez, índice de saliência, índice de abrangência e formal de copa. Os indivíduos de Curitiba prismatica apresentaram DAP médio de $15,91 \mathrm{~cm}$, com mínimo e máximo de 10,35 e 46,41 cm, respectivamente. A frequência de distribuição dos indivíduos foi assimétrica em relação à média. A altura total média foi de $8,98 \mathrm{~m}$ e a altura média de inserção da copa de 3,60 m, decorrendo que, em média, 60,05\% da altura de cada árvore é constituída pela copa. Devido à amplitude das medidas dos DAP e da altura total ocorreu alta variabilidade do grau de esbeltez, do índice de saliência e do índice de abrangência. A variável formal da copa indica que a espécie tem predomínio de copas arredondadas.
\end{abstract}

Accepted on

2020/03/02

Published in

2020/04/03

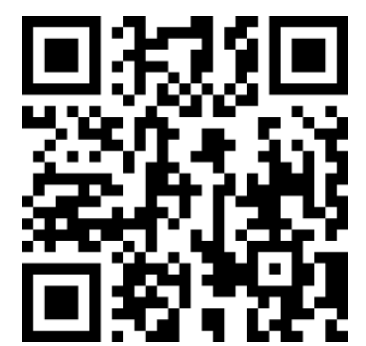

DOI:

http://dx.doi.org/10.34062/af s.v7i1.8150

\section{(cc) BY}

\section{Morphometric characterization of Curitiba prismatica}

\begin{abstract}
Morphometry and growth dynamics of Curitiba prismatica (D. Legrand) Salywon and Landrum were evaluated in a fragment of Mixed Araucaria Forest in Faxinal system, in Rebouças municipality, PR, Brazil. Trees with DBH $\geq 10 \mathrm{~cm}$ were included into analyses, which were performed by grouping and per diameter classes. The data were collected in 2017, when DBH, total height, crown insertion height, and the four crown projection rays were measured. Based on such information, the following morphometric traits were measured: crown diameter, crown length, crown ratio, degree of slenderness, salience index, crown coverage index, and crown formal. Curitiba prismatica presented mean DBH of $15.91 \mathrm{~cm}$, with a minimum and maximum of 10.35 and $46.41 \mathrm{~cm}$, respectively. The distribution frequency of the trees was asymmetric in relation to the mean. The mean of total height and the mean crown insertion height were $8.98 \mathrm{~m}$ and 3.60 $\mathrm{m}$, respectively, so that, on average, $60.05 \%$ height of each tree consisted of crown. Due to the range of DBH and total height measurements, a high variability was detected for the degree of slenderness, salience index and crown coverage index. The trait crown formal indicates that such a species has a predominance of rounded crowns.
\end{abstract}


Gardin et al.

\section{Introdução}

O estudo das características morfométricas de uma árvore e suas variáveis pode ser eficaz para avaliar as suas relações interdimensionais. Possibilitando assim pressupor o grau de competição de um povoamento e mensurar a estabilidade, vitalidade e produtividade de cada indivíduo (Durlo e Denardi 1998).

De acordo com Durlo e Denardi (1998), a altura total $(\mathrm{H})$, a altura de inserção da copa (HIC), o diâmetro a 1,3 m do solo (DAP) e o diâmetro da copa (DC) são as variáveis básicas na dedução das demais variáveis morfométricas da árvore. As principais variáveis morfométricas são comprimento da copa (CC), proporção de copa (PC), índice de saliência (IS), índice de abrangência (IA), formal de copa (FC) e grau de esbeltez (GE) (Durlo e Denardi 1998).

A razão entre o $\mathrm{DC}$ e a $\mathrm{H}$ da árvore é conhecida como índice de abrangência. Essa relação tende a diminuir com o incremento da altura da árvore, visto que o aumento na altura não é acompanhado na mesma proporção pelo aumento em diâmetro da copa (Tonini e Arcoverde 2005).

$\mathrm{O}$ GE é a relação entre a $\mathrm{H}$ e o DAP. Valores pequenos indicam que as árvores crescem mais em diâmetro do que em altura. Quando a variável GE apresenta valor superior a 100 é um indicativo que a árvore está suscetível a danos físicos, causados por ventos e tempestades, entre outros (Roveda et al. 2012).

A variável IS, que relaciona DC e o DAP, demonstra quantas vezes o diâmetro da copa é maior que o DAP da árvore. Essa relação pode então ser utilizada como indicador de desbaste e pode-se determinar, a qualquer tempo, o espaço a ser liberado ao redor de uma árvore selecionada, para que ela cresça sem concorrência. A variável FC expressa a relação entre o DC e o CC, caracterizando a forma geométrica da copa da árvore. A PC representa a porcentagem do $\mathrm{CC}$ em relação a $\mathrm{H}$ da árvore (Durlo e Denardi 1998).

O conhecimento da morfometria e da dinâmica de crescimento das formas das árvores torna-se necessário para aperfeiçoar as intervenções silviculturais. A necessidade desse conhecimento ganha maior importância principalmente quando se deseja utilizar espécies da flora nativa em programas de reflorestamento com finalidades econômicas (Durlo et al. 2004; Roman et al. 2009).

Curitiba. prismatica é uma espécie arbórea pertencente à família Myrtaceae. É uma espécie endêmica encontrada no sul do Brasil, ocorrendo no primeiro e segundo planaltos do Paraná e no planalto norte de Santa Catarina. Inicialmente classificada como Eugenia prismatica (D. Legrand), teve sua classificação definitiva em 2007 quando foi transferida para um novo gênero, Curitiba Salywon \& Landrum. Em sua área de ocorrência, frequentemente forma populações densas no sub- bosque da Floresta Ombrófila Mista FOM (Salymon e Landrum 2007).

Atualmente não existe na literatura estudo sobre a morfometria da espécie. Dessa forma, esse trabalho teve como objetivo avaliar as características morfométricas da Curitiba prismatica, e suas possíveis correlações.

\section{Material e Métodos}

Caracterização e localização da área de estudo

$\mathrm{O}$ estudo foi realizado em um fragmento de Floresta Ombrófila Mista (FOM) no município de Rebouças-PR, sendo a área localizada a uma latitude sul $25^{\circ} 40^{\prime} 12^{\prime \prime}$, e longitude oeste $50^{\circ} 32^{\prime} 56^{\prime}$ ', com uma altitude média de $815 \mathrm{~m}$ acima do nível do mar.

O clima regional é do tipo $\mathrm{Cfb}$, de acordo com a classificação de Köppen, apresentando estações climáticas bem definidas, com chuvas distribuídas durante todo o ano, com geadas severas e umidade relativa do ar acentuada. A precipitação média anual está entre 1500 e $1600 \mathrm{~mm}$. A temperatura média anual é de aproximadamente $18^{\circ} \mathrm{C}$, com mínima de $2^{\circ} \mathrm{C}$ e máxima de $32^{\circ} \mathrm{C}$.

O histórico da área consta de mais de um século de uso da floresta para extrativismo de produtos madeireiros (lenha, mourões, madeira em tora) e produtos não madeireiros, como a erva-mate e sementes, principalmente o pinhão. A atividade pastoril de bovinos, equinos, caprinos, suínos, entre outros, também é bastante difundida nas propriedades rurais.

Essa área encontra-se cadastrada, desde o ano de 1997, no Instituto Ambiental do Paraná - IAP, como Área de Uso Regulamentado - ARESUR. Após ser reconhecida formalmente como Unidade de Conservação, foi incluída no Cadastro Estadual de Unidades de Conservação (CEUC).

Nesse local, em 2012, foram instaladas e inventariadas 50 unidades permanentes, com o objetivo de avaliar o estado da FOM em Sistema Faxinal. Cada unidade permanente foi instalada com comprimento de $50 \mathrm{~m}$, no sentido norte-sul, e largura de $10 \mathrm{~m}$, no sentido leste-oeste, formando um retângulo de $500 \mathrm{~m}^{2}$ (Albuquerque 2015).

\section{Levantamento dos Dados}

Para o levantamento dos dados foram medidos, em abril de 2017, 225 indivíduos de Curitiba prismatica presentes nas unidades permanentes, que apresentaram DAP igual ou superior a $10 \mathrm{~cm}$. Os dados coletados foram o DAP, a $\mathrm{H}$, a HIC e os quatro raios de projeção de copa nos sentidos norte, sul, leste e oeste. A medida dos quatro raios foi utilizada para calcular o DC.

A identificação botânica e a catalogação da espécie foram realizadas no Herbário da Universidade Estadual do Centro-Oeste (HUCO), onde a exsicata foi depositada, recebendo o número de tombo 6303. 
As alturas das árvores foram medidas com o hipsómetro (Forestor Vertex III, SoilControl). Para as medições dos DAP e dos raios de copa utilizou-se fita métrica.

\section{Análise dos Dados}

Para a análise morfométrica foram avaliadas as variáveis: H, HIC, DAP, DC e as variáveis definidas por Burger (1939):

$C C=H-H I C$

$P C=C C / H \times 100$

$G E=H / D A P$

$I S=D C / D A P$

$I A=D C / H$

$F C=D C / C C$

Os dados foram analisados tanto de forma agrupada quanto estratificada por classes de

Tabela 1. Caracterização das variáveis morfométricas

\begin{tabular}{lccccc}
\multicolumn{6}{l}{ Tabela 1. Caracterização das variáveis morfométricas das árvores de Curitiba prismática } \\
\hline Variável & Mínimo & Máximo & Médio & Mediana & CV (\%) \\
\hline DAP (cm) & 10,35 & 46,41 & 15,91 & 14,42 & 32,76 \\
H (m) & 3,00 & 16,50 & 8,98 & 8,90 & 21,88 \\
HIC (m) & 1,32 & 8,50 & 3,60 & 3,20 & 36,90 \\
DC (m) & 1,95 & 12,10 & 4,80 & 4,65 & 33,16 \\
CC (m) & 0,70 & 13,80 & 5,38 & 5,30 & 34,16 \\
PC $(\%)$ & 8,79 & 87,89 & 59,71 & 61,82 & 24,27 \\
GE & 22,15 & 95,10 & 59,71 & 59,24 & 26,46 \\
IS & 11,32 & 71,91 & 31,32 & 30,30 & 32,83 \\
IA & 0,21 & 1,40 & 0,55 & 0,54 & 35,03 \\
FC & 0,34 & 2,20 & 0,98 & 0,90 & 43,87
\end{tabular}

Em que: DAP - diâmetro a 1,3 m do solo, $\mathrm{H}$ - altura total da árvore, HIC - altura de inserção da copa, DC diâmetro da copa, CC - comprimento da copa, PC - proporção da copa, GE - grau de esbeltez, IS - índice de saliência, IA - índice de abrangência, FC - formal da copa.

A alta variabilidade nos coeficientes de variação, principalmente das variáveis morfométricas HIC, IA e FC, provavelmente ocorreu devido às diferentes idades e estágios sucessionais, bem como aos diferentes níveis de concorrência em que a Curitiba prismatica estava submetida.

Conforme estudo realizado por Albuquerque (2015) nessa mesma área, foram encontradas 89 espécies, sendo a média de altura total de 9,76 m. A espécie Curitiba prismatica ocupava a décima diâmetro. Para definição do número de classes diamétricas (nc) utilizou-se a fórmula de Sturges:

$$
n c=1+3,3 \cdot \log n
$$

Em que: $\mathrm{n}=$ número de indivíduos observado.

A determinação do intervalo de classes foi realizada utilizando-se o resultado da razão entre a amplitude total e o nc.

As análises estatísticas foram realizadas com o software R, versão 3.2.2. As diferenças nas médias foram avaliadas pelo teste de Tukey, com nível de significância de $5 \%$.

\section{Resultados e discussão}

Características Morfométricas

$\mathrm{Na}$ área de estudo foram avaliados 225 indivíduos de Curitiba prismatica, sendo os resultados das variáveis morfométricas apresentados na Tabela 1. Estão dispostos os valores mínimos, máximos, médios, mediana e coeficiente de variação para as variáveis DAP, H, HIC, DC, CC, PC, GE, IS, IA e FC. 
Gardin et al.

abaixo do valor médio. Conforme Durlo et al. (2004), por se tratar de uma floresta inequiâneas, a grande amplitude de variação da HIC é esperada, visto que foram medidas árvores em diferentes idades e estágios sucessionais.

Estes valores de HIC observados para Curitiba prismatica são muito próximos aos resultados obtidos por Hellmann (2016), que encontrou HIC para Eugenia pyriformis Cambess valores variando de 1,10 a $7,30 \mathrm{~m}$, com média de $3,58 \mathrm{~m}$.

O DC dos indivíduos de Curitiba prismatica apresentou variação de 1,95 a 12,10 m, com média de 4,80 m e coeficiente de variação de 33,16\%. Tendo como base o valor médio observado de 4,80 m para a variável morfométrica DC (Tabela 1), pode-se supor que a espécie Curitiba prismatica necessita de uma área de $20 \mathrm{~m}^{2}$ para seu desenvolvimento. Com isso, a densidade máxima para o plantio da Curitiba prismatica, para não ter limitações em seu crescimento seria 400 ind. ha ${ }^{-1}$.

Homczinski (2017), em trabalho realizado na Floresta Nacional de Irati-PR (Flona de Irati), obteve para a Campomanesia xanthocarpa os valores médios de $14 \mathrm{~m}$ para H, 7,2 m para HIC e DAP de $32,09 \mathrm{~cm}$. Em relação à copa, a média para DC, CC e IA foram de 9,5 m 7,2 m e 0,66, respectivamente.

A média para PC observada na Curitiba prismatica (Tabela 1) foi de 59,71\%, com mínimo de $8,79 \%$ e máximo de $87,90 \%$. O coeficiente de variação para essa variável morformétrica foi de 24,27\%. Segundo Roman et al. (2009), essa amplitude para a PC é resultado das diferentes idades e graus de concorrência a que as árvores estão submetidas.

Segundo Condé et al. (2013), espécies com elevada proporção de copa, como é o caso da Curitiba prismatica, apresentam a maior parte de sua estrutura em forma de copa, estando assim altamente dependente da radiação solar para realizar sua manutenção e desenvolvimento. Além disso, essa característica pode ser desejável para produtos não madeireiros, derivados de uma copa exuberante (sementes, frutos, folhas etc.). Porém, por outro lado, para produtos com fins madeireiros alta $\mathrm{PC}$ pode não ser adequado (Tonini et al. 2008; Condé et al. 2013).

Os valores encontrados nesse trabalho para PC são semelhantes aos dados de Orellana e Koehler (2008) levantados em um fragmento de FOM. Neste estudo citado, foi obtida uma média de PC igual a $55,2 \%$, em que as árvores analisadas também tinham DAP igual ou superior a $10 \mathrm{~cm}$.

A variável morfométrica GE para a Curitiba prismatica apresentou valor mínimo de 22,15 e máximo de 95,10, com média de 59,71. A amplitude das medidas dos DAP e da altura total resultou em uma alta variabilidade do GE, cujo coeficiente de variação foi de $26,46 \%$.

Lanzarin (2016) afirma que altos valores, maiores que 100, para GE podem ser resultados da falta de luz devido à competição entre as árvores, ocasionando a inibição do crescimento em diâmetro. Indivíduos com estas características apresentam troncos finos e altura elevada, o que pode levar a sua instabilidade, principalmente no caso de fortes ventos. Dessa forma, os valores para o GE observado na Curitiba prismatica indicam, que na área de estudo, a maioria dos indivíduos da espécie não se encontra suscetível a danos físicos, causados principalmente por ventos.

Os valores de IS observados indicam que em média as copas da Curitiba. prismatica são 31,32 vezes maiores que o DAP. Essa relação pode ser utilizada como indicador de produção para a espécie, sendo que as espécies de maior crescimento em termos de volume de madeira apresentam valores entre 15 e 20 para o IS. Espécies com valores de IS superiores a 20 tendem a um baixo crescimento e valores acima de 40 indicam baixa produção (Tonini e Arco-verde 2005). Assim, conforme os valores observados na Tabela 1 para a variável IS, a espécie Curitiba prismatica pode ser considerada uma espécie de baixo crescimento.

O IA apresentou valores mínimo e máximo de 0,21 e 1,40, respectivamente. Observou-se que o IA apresentou a tendência em diminuir com o aumento da altura para todas das árvores. Conforme Tonini e Arco-verde (2005), isso ocorre devido ao crescimento em altura ser maior que o crescimento do diâmetro de copa.

Para a variável FC foi observada a maior variabilidade para as variáveis morfométricas analisadas. Os valores variaram entre 0,34 e 2,20, com média de 0,98 e coeficiente de variação de $43,87 \%$. De acordo com esses resultados encontrados para FC da Curitiba prismatica é possível indicar que na comunidade existem os três tipos de copas: copas alongadas (FC abaixo de 1), copas arredondas (FC próxima a 1) e copas chatas (FC acima de 1). Porém, levando em consideração a média dos resultados, $(1,03)$ a tendência dos indivíduos de Curitiba prismatica é ter copas arredondadas.

\section{Características morfométricas por classes} diamétricas

As frequências por distribuição diamétrica dos indivíduos de Curitiba prismatica, bem como de todas as espécies da área de estudo, estão representadas na Figura 1. As classes diamétricas acima de $35 \mathrm{~cm}$, por apresentarem frequência inferior a um indivíduo, foram suprimidas das análises estatísticas posteriores.

De acordo com a Figura 1, observa-se que a classe diamétrica de 10-15 cm é formada por 308,57 ind. ha $^{-1}$, incluindo todas as espécies encontradas na área de estudo. Desse total, a Curitiba prismatica, com 90,71 ind. ha $^{-1}$, representa $29,40 \%$ de todas as árvores. $\mathrm{Na}$ classe diamétrica de $15-20 \mathrm{~cm}$ a ocorrência da Curitiba prismatica foi relativamente 
maior, representando 38,82\% de todos os indivíduos inventariados. Nas classes diamétricas acima de 20 cm a participação relativa da Curitiba prismatica sofre gradativa redução, até não mais ocorrer nas classes acima de $50 \mathrm{~cm}$ de DAP.

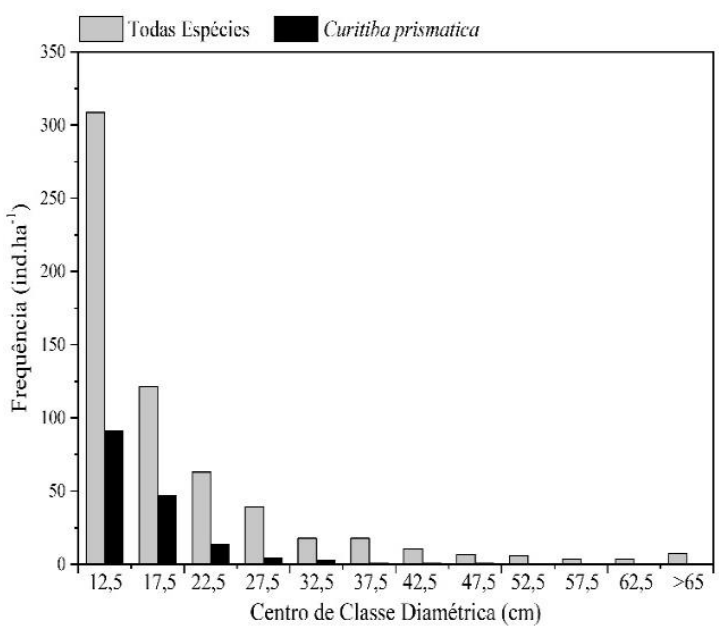

Figura 1. Histograma das frequências dos DAP de Curitiba prismatica avaliadas em 2017, em Rebouças-PR

O maior DAP encontrado para Curitiba prismatica foi de $46,41 \mathrm{~cm}$, indicando ser uma característica da espécie o crescimento diamétrico limitado a essa faixa de valores. Embora a espécie não esteja presente nas classes diamétricas acima de $50 \mathrm{~cm}$, observa-se que a sua distribuição diamétrica apresentou o formato de "J" invertido, característico da estrutura diamétrica em florestas naturais.

Neste tipo de estrutura, há uma maior concentração de indivíduos nas menores classes, com redução progressiva da frequência conforme o aumento de classe. Essa distribuição indica a ocorrência de regeneração e estabilidade na recomposição florestal da espécie (Meyer 1952; Dalla Lana et al. 2013; Reis et al. 2014).

As Figuras 2 e 3 apresentam as variáveis morfométricas observadas, divididas por classes diamétricas. Na Figura 2 estão representadas as variáveis $\mathrm{H}, \mathrm{HIC}, \mathrm{DC}, \mathrm{CC}$ e PC, enquanto na Figura 3 as variáveis GE, IS, IA e FC.

As variáveis $\mathrm{H}$ e HIC apresentaram um comportamento semelhante, aumentando à medida que ocorreu incremento do DAP. Para as classes diamétricas de 10 a $25 \mathrm{~cm}$ não ocorreram diferenças significativas. Para as classes diamétricas que englobam os diâmetros acima de $25 \mathrm{~cm}$ observaramse diferenças significativas nessas variáveis, conforme Figura 2A e 2B. Isso pode indicar que a Curitiba prismatica está dividida em dois estratos arbóreos, sendo as árvores com mais de $10 \mathrm{~m}$ pertencente ao primeiro estrato e as abaixo dessa medida, compondo o segundo estrato. De acordo com a Figura 2C, a variável DC para a classe diamétrica de 30-35 cm difere significativamente das demais classes. Essa classe é a que possui maior $\mathrm{H} \mathrm{e}$ conforme observado à campo, elas fazem parte do dossel da floresta.

As variáveis morfométricas CC e PC, Figura $2 \mathrm{D}$ e $2 \mathrm{E}$, não diferiram em nenhuma das classes diamétricas analisadas. Isso indica que essas variáveis se mantêm constante com o aumento do DAP. Provavelmente, essa é uma característica da espécie e pouco afetada com as condições do ambiente, como luminosidade e competição.

As classes diamétricas, Figura 3A, apresentaram valores decrescentes para o GE, sendo que as classes de $10-15 \mathrm{~cm}$ e de $15-20 \mathrm{~cm}$ diferiram significativamente das demais. As classes acima de $20 \mathrm{~cm}$ não se diferenciaram, indicando estabilidade entre o crescimento em altura e o DAP.

As árvores da classe diamétrica 10-15 cm possuem o maior valor para GE. Conforme Roveda et al. (2012), isso indica que essas árvores são as mais suscetíveis a danos físicos, como vento e tempestades. Provavelmente esses indivíduos buscam primeiramente alcançar o dossel, onde há mais energia luminosa, e após isso, aumentam o DAP.

Para as variáveis IS, IA e FC, Figura 3B, 3C e 3D respectivamente, não foram observadas diferenças significativas entre as classes diamétricas. Além da grande variabilidade dos dados, que contribuiu para não ter ocorrido diferenças significativas, isso pode demonstrar que essas são também características da própria espécie e são pouco alteradas pelas características de competição da floresta.

Os valores apresentados pela variável morfométrica FC, devido à grande variabilidade, não apresentou diferenças significativas entre as classes diamétricas. Dessa forma, conforme Roman et al. (2009), pode-se classificar a forma das copas da Curitiba prismatica, independente do diâmetro da árvore, como tendo majoritariamente a forma arredonda.

De forma geral, as variáveis observadas para a Curitiba prismatica apresentaram grande variabilidade, principalmente a variável FC. De acordo com Lanzarin (2016), essa grande amplitude nos valores é resultado da plasticidade que a espécie tem em relação à sua copa, adaptando-se às diferenças impostas pelo ambiente e demais espécies. 

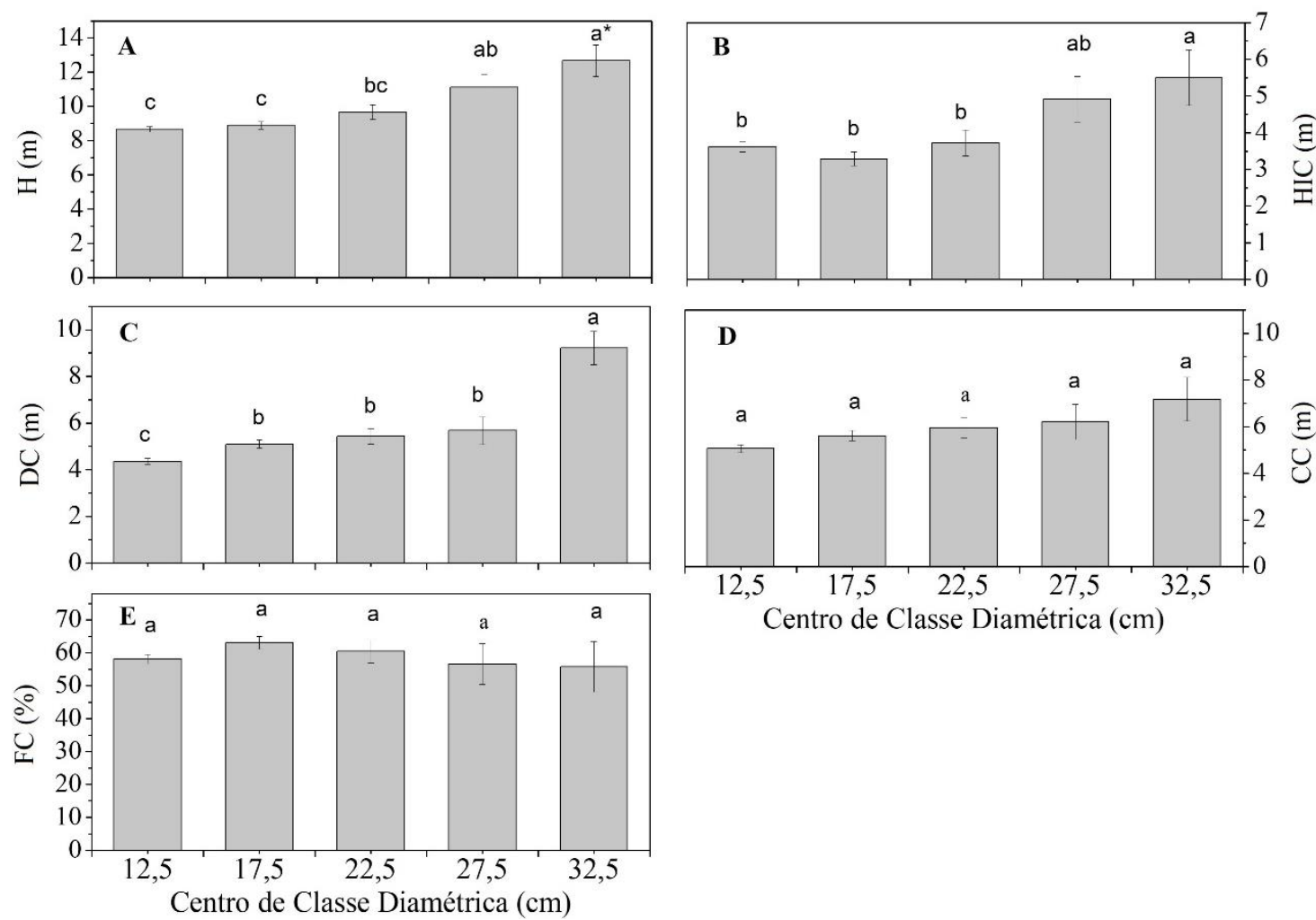

Figura 2. Variáveis morfométricas: altura total (A), altura de inserção da copa (B), diâmetro da copa (C), comprimento da copa (D) e proporção copa (E), da Curitiba prismatica, por classes diamétricas.

* Médias seguidas pela mesma letra não se diferenciam entre si pelo teste de Tukey a 5\% de probabilidade.
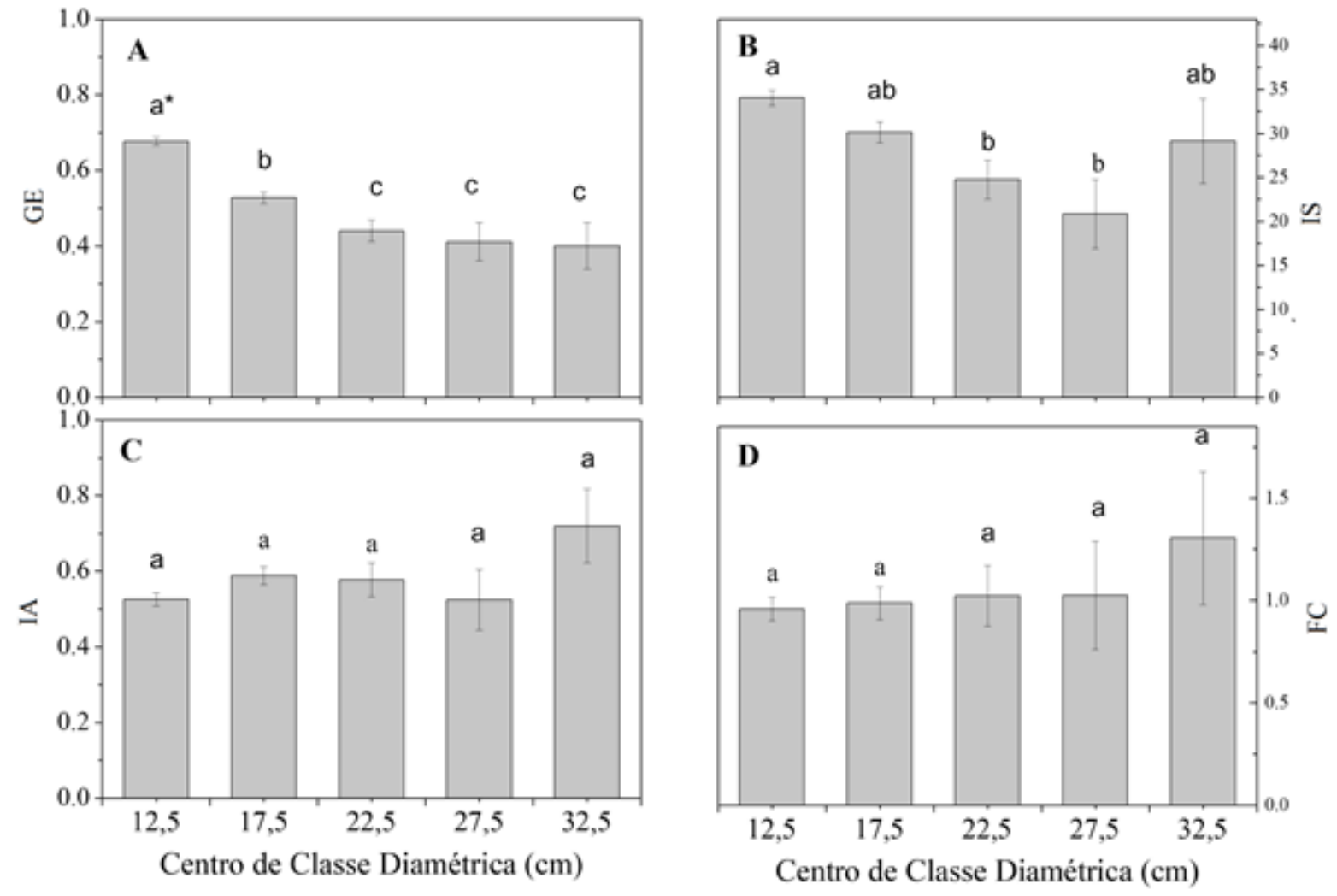

Figura 3. Variáveis morfométricas: (A) grau de esbeltez, (B) índice de saliência, (C) índice de abrangência e (D) formal da copa, da Curitiba prismatica por classes diamétricas.

* Médias seguidas pela mesma letra não se diferenciam entre si pelo teste de Tukey a 5\% de probabilidade. 
Gardin et al.

\section{Conclusão}

A grande variabilidade apresentada pelas variáveis biométricas e morfométricas estuadas indica que Curitiba prismatica possui plasticidade adaptativa. Isso foi observado principalmente em relação à copa, em virtude da ampla variação dos raios da copa observados. Devido a essa variabilidade, as variáveis morfométricas apresentaram baixa correlação.

A distribuição diamétrica indica que, na área estudada, a espécie está conseguindo se regenerar e possui estabilidade na sua recomposição, mesmo a espécie estando sujeita a perturbações antrópicas decorrentes do uso da área (Sistema Faxinal).

$\mathrm{Na}$ área de estudo verificou-se que os indivíduos analisados possuem limite máximo em torno de $50 \mathrm{~cm}$ de DAP. Outros estudos devem ser realizados em diferentes áreas, sob diferentes manejos, para determinar se este é o limite máximo de DAP para Curitiba prismatica.

\section{Referencias}

Albuquerque JM (2015) Fatores ecológicos e ocorrência de espécies na floresta ombrófila mista, em sistema faxinal. Tese, Universidade Federal do Paraná. 93p.

Burger H (1939) Baumkrone und zuwachs in zwei hiebsreifen fichtenbeständen. Mitteilungen der Schweizerischen Anstalt für das Forstliche Versuchswesen, 21:147-176.

Condé TM, Lima MLM, Lima Neto EM, Tonini H (2013) Morphometric of four species in agroforestry systems in the municipality of Porto Velho, Rondônia. Agroambiente On-line, 7(1):18-27. doi: 10.18227/1982-8470ragro.v7i1.932

Dalla Lana M, Brandão CFLS, Péllico Neto S, Marangon LC, Retslaff FAS (2013) Distribuição diamétrica de Escheweilera ovata em um fragmento de Floresta Ombrófila Densa - Igarassu, PE. Floresta, 43(1):59-68. doi: 10.5380/rf.v43i1.25252

Durlo MA, Denardi L (1998) Morfometria de Cabralea canjerana, em mata secundária nativa do Rio Grande do Sul. Ciência Florestal, 8(1):55-66.

Durlo MA, Sutili FJ, Denardi L (2004) Modelagem da copa de Cedrela fissilis Vellozo. Ciência Florestal, 14(2):79-89.

Hellmann SS (2016) Ecofisiologia e morfometria como ferramenta para o manejo sustentável de Eugenia pyriformis. Dissertação, Universidade Estadual do Centro-Oeste. 90p.

Homczinski I (2017) Distribuição espacial, dinâmica e biometria de Campomanesia xanthocarpa (Mart.)
O. Berg. em Floresta Ombrófila Mista. Dissertação, Universidade Estadual do Centro-Oeste, Irati. 88p.

Lanzarin K (2016) Relações morfométricas e potencial de manejo de Maclura tinctoria (L.) D. Don Ex. Steud em formações secundárias do noroeste do rio Grande do Sul. Dissertação, Universidade Federal de Santa Maria. 77p.

Meyer HA (1952) Structure, growth, and drain in balanced uneven-aged forests. Journal of Forestry, 52(2):85-92.

Orellana E, Koehler AB (2008) Relações morfométricas de Ocotea odorifera (Vell.) Rohwer. Revista Acadêmica Ciências Agrárias e Ambientais, 6(2):229-237.

Reis LP, Ruschel AR, Silva JNM, Reis PCM, Carvalho JOP, Soares MHM (2014) Dinâmica da distribuição diamétrica de algumas espécies de Sapotaceae após exploração florestal na Amazônia Oriental. Revista de Ciências Agrárias, 57(3):234243. doi: $10.4322 /$ rca.ao 1401

Roman M, Bressan DA, Durlo MA (2009) Variáveis morfométricas e relações interdimensionais para Cordia trichotoma (Vell.) Arráb. Ex Steud. Ciência Florestal, 19(4): 473-480. doi: $10.5902 / 19805098901$

Roveda M, Dalgallo B, Dias NA, Figueiredo Filho A, Muller CS (2012) Morfometria de Araucaria angustifolia (Bertol.) Kuntze na Floresta Nacional de São Francisco de Paula, RS. In: IV Congresso Florestal Paranaense, Curitiba, Brasil.

Salywon AM, Landrum LR (2007) Curitiba (Myrtaceae): A new genus from the planalto of southern Brazil. Brittonia, 59(4):301-307. doi: 10.1663/0007-

196X(2007)59[301:CMANGF]2.0.CO;2

Tonini H, Arco-Verde MF (2005) Morfologia da copa para avaliar o espaço vital de quatro espécies nativas da Amazônia. Pesquisa Agropecuária Brasileira, 40(7):633-638. doi: 10.1590/S0100204X2005000700002

Tonini H, Kaminski PE, Costa P (2008) Relação da produção de sementes de castanha-do-brasil com características morfométricas da copa e índice de competição. Pesquisa Agropecuária Brasileira, 43(11):1509-1516. doi: 10.1590/S0100204X2008001100009 